\title{
PAROXYSMAL NOCTURNAL HEMOGLOBINURIA IN CHILDHOOD: AN UNCOMMON PRESENTATION
}

\author{
V. GUPTA, V. TILAK*, S. V. K. RAMAKRISHNA, B. D. BHATIA
}

\section{ABSTRACT}

\begin{abstract}
An eight year old boy presented with severe anemia and bleeding spots. Complete blood count showed pancytopenia. There was mild reticulocytosis. Bone marrow was hypocellular with normoblastic erythroid hyperplasia. Ham's test (acidified serum test) was positive which confirmed the diagnosis of Paroxysmal nocturnal hemoglobinuria (PNH). Although PNH is rare in childhood, it should be considered as a diagnostic possibility in cases of aplastic anemia as the two conditions can coexist. The presence of PNH in association with aplastic anemia can influence the outcome of the latter.
\end{abstract}

Key words: Paroxysmal nocturnal hemoglobinuria, aplastic anemia, pancytopenia

\section{INTRODUCTION}

Paroxysmal nocturnal hemoglobinuria (PNH) is an acquired clonal hematologic disorder which has been traditionally classified as hemolytic anemia. However, it is also associated with a component of bone marrow failure and a liability for venous thrombosis. It is a unique disorder characterized by a triad of hemolytic anemia, pancytopenia and thrombosis. ${ }^{[1]}$ There can be either sequential or concurrent appearance of aplastic anemia and $\mathrm{PNH}$ in an individual patient. Presence

Departments of Pediatrics and *Pathology Institute of Medical Sciences, Banaras Hindu University, Varanasi - 221 005, India

\section{Correspondence}

Vineeta Gupta

Department of Pediatrics, Institute of Medical Sciences,

B.H.U, Varanasi 221005 , India

E-mail: sanjeevggupta@sancharnet.in of PNH type cells in cases of bone marrow failure syndromes suggests a relatively benign nature of the disease. ${ }^{[2]} \mathrm{An}$ attempt should be made to identify the condition before starting therapy. Majority of the cases occur in adulthood with isolated case reports in children. ${ }^{[3,4]}$ An extensive literature search revealed only one case report from India in a child ${ }^{[5]}$ which prompted us to report an interesting case of severe anemia in an eight year old boy which turned out to be $\mathrm{PNH}$ with aplastic anemia.

\section{CASE REPORT}

An eight year old boy presented to Pediatric out-patient department in January 2004 with the complaints of progressively increasing pallor and fatigability for 2 months and bleeding spots all over the body for 1 month duration. On, examination, the child had 
severe pallor with petechial and purpuric spots all over the body. The liver was palpable $2 \mathrm{~cm}$ below the costal margin and spleen was $1 \mathrm{~cm}$. There was no significant lymphadenopathy. On investigation, the hemoglobin was $6.2 \mathrm{gm} \%$, total leucocyte count was $3550 / \mathrm{mm}^{3}$ with a differential count of $38 \%$ neutrophils, $2 \%$ eosinophils and $60 \%$ lymphocytes. The platelet count was $18000 /$ $\mathrm{mm}^{3}$. There were no abnormal cells in the peripheral smear. Bone marrow aspiration was hypocellular with normoblastic erythroid hyperplasia. The trephine biopsy showed decreased cellularity. The leucopoeisis and thrombopoeisis were depressed with a myeloid to erythroid ratio of $1.5: 1$. The reticulocyte count was $2.5 \%$ with a negative Coomb's test. The serum LDH was 700 IU/ L. Serum bilirubin was within normal limits and urine examination was negative for hemoglobinuria. In view of the normoblastic erythroid hyperplasia in the bone marrow and reticulocytosis against a backdrop of pancytopenia, a diagnosis of Paroxysmal nocturnal hemoglobinuria was considered. Ham's test (acidified serum test) was carried out which was positive. The final diagnosis was $\mathrm{PNH}$ with aplastic anemia. The child was given supportive treatment with packed red cells and platelet transfusion and was started on oral cyclosporine $A$ alone as he could not afford treatment with antithymocyte globulin. Two months in follow up, the child continued to have anemia and pancytopenia but there was some improvement in the thrombocytopenia. The platelet count rose to $46,000 / \mathrm{mm}^{3}$. Unfortunately, the child was lost to follow up subsequently.

\section{DISCUSSION}

PNH was first described a century ago but the molecular defects have been identified only recently. Because of a somatic mutation in the phosphatidyl inositol glycan complementation group-A (PIG-A) gene, a number of proteins such as membrane inhibitor of reactive lysis (MIRL, CD59) and decay accelerating factor (DAF, CD55) are absent from the surface of red blood cells..$^{[1]}$ This renders the cells susceptible to lysis by complement resulting in intravascular hemolysis and hemoglobinuria. The hemoglobinuria may be intermittent on awakening in the morning or may be continuous throughout the day. However, some patients may never develop it.

$\mathrm{PNH}$ is a disease of adults presenting in $3^{\text {rd }}$ to $5^{\text {th }}$ decade. In a large series of 78 cases of $\mathrm{PNH}$ diagnosed in 10 years, the mean age of diagnosis was 34 years. ${ }^{[6]}$ In the largest series of 26 cases of $\mathrm{PNH}$ with onset in childhood the age ranged from 8 months to 21 years and only $3.8 \%$ cases were younger than 10 years. ${ }^{[7]}$ There was significant difference between young patients with $\mathrm{PNH}$ and adult patients. Hemoglobinuria as the presenting complain was seen in only $15 \%$ of children as against $50 \%$ in adults. In contrast, bone marrow failure was much more common in young than in the adults (58\% versus $25 \%$ ).

PNH and aplastic anemia are clinically related. The disorders may present simultaneously or one may evolve into another. The cause of bone marrow failure in $\mathrm{PNH}$ is not very clear. The possible explanations are that $\mathrm{PNH}$ clone suppresses the normal marrow progenitors or it has an intrinsic growth and proliferative advantage compared to normal stem cells. ${ }^{[1]}$ The high prevalence of bone marrow failure frequently leads to an initial diagnosis of aplastic anemia rather than $\mathrm{PNH}$ as happened in the present case. He was initially diagnosed as a case of aplastic anemia but because of the presence of normoblastic erythroid hyperplasia in the bone marrow and mild reticulocytosis, the possibility of $\mathrm{PNH}$ was thought of. The reticulocyte count in these cases is not parallel with the extent of hemolysis and this reflects the underlying bone marrow failure which always coexists with $\mathrm{PNH} .{ }^{[1]}$ Venous thrombosis is the third component of this condition, the pathophysiology of which is not very clear The possible reasons are impaired fibrinolysis, hypercoagulability and hyperactivity of platelets because of the lack of CD59 on PNH platelets. However, thrombotic complication was not present in our case.

Ham's test is a highly sensitive test for the diagnosis of $\mathrm{PNH}$. It may miss the diagnosis initially if the PNH clone is small. Therefore it is important to repeat the Ham's test at regular intervals to avoid missing the diagnosis. The availability of flow-cytometry has further improved the diagnosis of $\mathrm{PNH}$. In the series of 78 cases of PNH by Zhao et $a^{\left[{ }^{[6]}\right.}$ Ham's test was positive in $65.8 \%$ cases whereas CD59 and CD55 were found deficient in $100 \%$ of cases.
Treatment of the condition depends upon the clinical presentation. In patients presenting with the features of aplastic anemia, either cyclosporine (Cy A) alone or in combination with antithymocyte globulin (ATG) have been tried. Results of a combination treatment (ATG + Cy A) are better than Cyclosporine alone. ${ }^{[8,9]}$ Allogenic haemopoietic cell transplantation has been tried successfully. ${ }^{[10]}$ In the present case cyclosporine was used alone because of financial constraints. In cases with recurrent hemolytic episodes short courses of oral prednisolone have been tried around the time of hemolysis. ${ }^{[1]}$

\section{REFERENCES}

1. Luzzatto L, Notaro R. Paroxysmal nocturnal hemoglobinuria. In: Handin RI, Lux SE, Stossel TP editors. Blood: Principles and Practice of Hematology. Philadelphia; Lippincott Williams \& Wilkins: 2003. p. 318-32.

2. Wang $\mathrm{H}$, Chuhjo $\mathrm{T}$, Yasue $\mathrm{S}$, Omine M, Nakao S. Clinical significance of a minor population of $\mathrm{PNH}$ type cells in bone marrow failure syndrome. Blood 2002;100:3897-902.

3. Dolzel Z, Dostalkova D, Blatny J, Starha J, Gerycova H. Paroxysmal nocturnal hemoglobinuria in a girl with hemolysis and "hematuria". Pediatr Nephrol 2004;19:1177-9.

4. Wainright L, Brodsky RA, Eranus LK, Poyiadjis S, Naidu G, Mckinnon D. Paroxysmal nocturnal hemoglobinuria arising from Fanconi anemia. J Pediatr Hematol Oncol 2003;25:167-8.

5. Yaranal PJ, Basu D, Narayanan P, Mahadevan S. Paroxysmal nocturnal hemoglobinuria with onset in childhood: a case report. Indian J Pathol Microbiol 2004;47:233-5.

6. Zhao M, Shao Z, Li K, Chen G, Liu H, Zhang Y. Clinical analysis of 78 cases of paroxysmal nocturnal hemoglobinuria diagnosed in past 10 
years. Chin Med J 2002;115:398-402.

7. Ware RE, Hall SE, Rosse WF. Paroxysmal nocturnal hemoglobinuria with onset in childhood and adolescence. New Eng J Med 1991;325:9915.

8. Ebenbichler CF, Wurfzner R, Sandhofer AD, Niederweiser D, Dierich MP, Patsch JR. Anti thymocyte globulin treatment of a patient for $\mathrm{PNH}$ haemoglobinuria- aplastic anemia syndrome: complement activation and transient decrease of $\mathrm{PNH}$ clone. Immunobiology 1996;196:513-21.

9. Saso R, Marsh J, Cevreska L, Szer J, Gale RP, Rowlings PA, et al. Bone marrow transplantation for PNH. Br J Haematol 1999;104:392-6.

10. Lee JL, Lee JH, Lee JH, Choi SJ, Kim S, Seol M. Allogenic hematopoetic cell transplantation for paroxysmal nocturnal hemoglobinuria. Eur $\mathrm{J} \mathrm{Hematol}$ 2003;71:114-8. 